\title{
Tendencias en Investigación y Aplicaciones Prácticas de la Ingeniería de Software: Una Selección del CONISOFT 2015
}

\author{
Raúl A. Aguilar Vera ${ }^{1}$, Reyes Juárez Ramírez ${ }^{2}$, Antonio A. Aguileta Güemez ${ }^{1}$ \\ 1. Cuerpo Académico de Tecnologías para la Formación en Ingeniería de Software. Facultad de Matemáticas, Universidad \\ Autónoma de Yucatán. Mérida 97000, México \\ 2. Facultad de Ciencias Químicas e Ingeniería, Universidad Autónoma de Baja California. Tijuana, 22390, México \\ avera@uady.mx,reyesjua@uabc.mx, aaguilet@uady.mx
}

\begin{abstract}
Resumen - En el presente apartado se presenta una sinopsis del Congreso Internacional de Investigación e Innovación en Ingeniería de Software, celebrado en la ciudad de San Luis Potosí, México, los días 27, 28 y 29 de abril de 2015. En este documento los editores invitados presentan los títulos y resúmenes de cinco trabajos seleccionados para este número especial de la Revista Latinoamericana de Ingeniería de Software.
\end{abstract}

\section{Palabras clave- CONISOFT, Ingeniería de Software, REDMIS.}

\section{INTRODUCCION}

La Ingeniería de Software (IS) como disciplina profesional tiene pocas décadas de existencia, el término "Ingeniería de Software" fue propuesto por Friedrich L. Bauer en una reunión del Comité de Ciencia de la Organización del Tratado del Atlántico Norte (OTAN) celebrada en otoño de 1967. En ese entonces se discutía la creciente complejidad que el proceso de desarrollo de software presentaba - disciplina conocida en aquella época como Programación. La problemática antes citada fue bautizada como la "Crisis del Software", la cual dio origen a un par de conferencias auspiciadas por la OTAN entre 1968 y 1969, en las que se sentaron las bases para diversos aspectos vinculados con la naciente disciplina (Naur y Randell, 1969; Buxton y Randell, 1970).

El proceso de construcción software como disciplina ingenieril, requiere de buenas prácticas, metodologías, técnicas $\mathrm{y}$ herramientas que únicamente pueden ser obtenidos $\mathrm{y}$ validados mediante la investigación, desarrollo e innovación tecnológica. En este sentido, el Congreso Internacional de Investigación e Innovación en Ingeniería de Software (CONISOFT) desde su primera edición, ha promovido el establecimiento de estrategias dentro de la academia que permitan lograr fortalezas y promover el progreso en la investigación y la docencia de la disciplina de IS a nivel nacional e internacional.

En la edición del CONISOFT'15, realizada en la ciudad de San Luis Potosí, México, del 27 al 29 de abril, se recibieron treinta y dos artículos de investigación provenientes de veinte instituciones de México, Perú y Colombia, de los cuales, el Comité Técnico Internacional, integrado por miembros de siete países: México, España, Argentina, Colombia, Uruguay, Estados Unidos y Brasil, seleccionó a los veinte trabajos mejor evaluados.

En este número especial se presentan versiones extendidas de cinco trabajos seleccionados que fueron presentados en las mesas de trabajo del evento. En las siguientes secciones se presentan los títulos, autores, instituciones y resúmenes de los cinco trabajos antes citados.

\section{MÉTODO BASADO EN INGENIERÍA INVERSA PARA LA REFACTORIZACIÓN DE BASES DE DATOS.}

Los autores Jessica Mariana Villar García, Miguel Ehécatl Morales-Trujillo y Guadalupe Ibargüengoitia-González, son integrantes del Grupo de Investigación KUALI-KAANS de la Universidad Nacional Autónoma de México.

Resumen: Las bases de datos son un componente esencial de los sistemas de software. La criticidad de las bases de datos ha ido en aumento debido a la relevancia que tienen los datos en el contexto tecnológico actual. Por tal razón, los datos almacenados en ellas tienen que ser precisos, consistentes y respetar ciertas reglas de integridad para que al ser interpretados provean información de calidad. Las características anteriores se logran con un buen diseño; sin embargo, no todas las bases de datos cuentan con uno. El artículo presenta a $\rho \mathrm{DB}$, un método de ingeniería inversa para refactorizar el diseño e implementación de bases de datos relacionales inmersas en sistemas de software en operación; su validación se llevó a cabo mediante un caso práctico, cuyos resultados permitieron establecer la utilidad y pertinencia del método, así como sus oportunidades de mejora.

\section{SISTEMA DE RECOMENDACIÓN PARA LA BÚSQUEDA PERSONALIZADA EN UN REPOSITORIO DE TRABAJOS DE TITULACIÓN.}

Los autores Rodrigo Vences Nava, Víctor Hugo Menéndez Domínguez y Alfredo Zapata González, son investigadores de la Universidad Autónoma de Yucatán.

Resumen: La producción académica de estudiantes en una institución educativa reúne resultados de investigaciones actuales y originales de diversas temáticas. Hoy en día podemos encontrar repositorios de tesis digitales en Internet disponibles para su consulta. Se han hecho esfuerzos por estandarizar dichos repositorios para establecer interoperabilidad entre diferentes instituciones; Sin embargo estos esfuerzos se centran más en la recuperación de información que en la recomendación de contenido. El modelo que se propone en el artículo es una solución para la búsqueda, recuperación y recomendación de trabajos de titulación en una institución educativa, personalizando la recuperación de información utilizando filtrados por contenido y colaborativo. El sistema de recomendación resultante, producto de esta investigación, se espera facilite el acceso de forma particular a 
los trabajos de titulación del área de ciencias sociales tanto a profesores como estudiantes permitiendo realizar consultas sobre temas específicos, recuperando la información solicitada y personalizándola de acuerdo al perfil de usuario construido con base en su interacción con el sistema.

\section{ANALIZANDO LA MANTENIBILIDAD DE SOFTWARE DESARROLLADO DURANTE LA FORMACIÓN UNIVERSITARIA.}

Los autores Héctor Pérez-González, Francisco Martínez Pérez, Sandra Nava Muñoz, Alberto Núñez Varela, Miriam Vázquez Escalante y Antonio Flores Saucedo, provienen de la Universidad Autónoma de San Luis Potosí.

Resumen: La calidad de los programas de software (SW) es considerada cuando éstos se realizan bajo estrictas metodologías y siguiendo estándares que intentan reducir la probabilidad de presentar defectos. La obtención de métricas de SW es un método útil para minimizar dicha probabilidad. Para evaluar la calidad de un programa de SW, uno de los indicadores más importantes es la mantenibilidad, la cual se puede medir mediante la obtención de un conjunto de métricas. La mantenibilidad es la facilidad con la que un sistema de SW puede ser modificado y es un atributo que afecta de manera crucial al costo de desarrollo del mismo. La literatura muestra estudios sobre mantenibilidad y la alteración de este parámetro cuando los programas evolucionan. El trabajo muestra los resultados de analizar 315 programas realizados por estudiantes universitarios de carreras en Ingeniería en Computación e Informática y la evolución que la mantenibilidad y otras métricas básicas presentan en función del progreso en la formación académica de sus autores.

\section{APLICANDO LA METODOLOGÍA PSP PARA EL DESARROLLO DE UN SISTEMA DE RECONOCIMIENTO DE GESTOS.}

Los autores Erik Ramos Pérez, Carlos Alberto Fernández y Fernández, así como Edwin León Hernández, realizan investigación auspiciados por la Universidad Tecnológica de la Mixteca.

Resumen: MUDI es un sistema reconocedor de gestos, capaz de detectar el acercamiento, rotación y cambiar de pieza arqueológica modeladas en 3D existentes en el MureH. El sistema está contextualizado para un museo interactivo en el que los usuarios puedan descubrir las características de las piezas de una forma entretenida y divertida. Para desarrollar el sistema se utilizó PSP, el cual es una metodología encaminada al proceso personal, establecido y regido por etapas bien establecidas, una de ellas es el diseño que permite tener una buena estructura del sistema traduciéndose en una fácil implementación y control de los errores de codificación, así como el manejo de los requerimientos. Para medir la interacción, efectividad y facilidad de uso de MUDI se usaron técnicas de usabilidad, obteniendo resultados favorables, específicamente el acercamiento tuvo una calificación de 4.8, rotación de 4.0 y cambiar pieza de 4.4 en una escala del 0 al 5 .

\section{ESPECIFICACIÓN DE REQUERIMIENTOS PARA EL Desarrollo de Software Automotriz en MéXico.}

Los autores Jorge Aguilar Cisneros y Carlos Alberto Fernández y Fernández, son investigadores de la Universidad Popular Autónoma del Estado de Puebla, y de la Universidad Tecnológica de la Mixteca, respectivamente.

Resumen: Actualmente, dentro del sector automotriz, México es reconocido a nivel mundial, como un excelente país ensamblador, sin embargo, países como Alemania, Estados Unidos y Japón, entre otros, son reconocidos mundialmente además de ensambladores como países innovadores de éste sector. Bajo este escenario, se propone que México incursione en la investigación e innovación automotriz, particularmente, en el área de desarrollo de sistemas de software automotriz embebido tanto críticos como no críticos. Para llevar a cabo la propuesta antes citada, en el artículo se presenta la situación del desarrollo de software automotriz en México, así como una revisión de los principales modelos de ciclo de vida, estándares y herramientas utilizadas en la construcción de sistemas de software automotriz embebido. De manera particular, se presenta la especificación de requerimientos para el sector automotriz, como un área inicial de atención.

\section{AGRADECIMIENTOS}

Agradecemos a los autores de los cinco artículos seleccionados el esfuerzo por generar versiones extendidas para integrar este número especial de la Revista Latinoamericana de Ingeniería de Software; esperamos con este esfuerzo - en colaboración con la Red de Ingeniería de Software de Latinoamerica (RedISLA) - contribuir en mejorar la visibilidad de los trabajos presentados por dichos autores en el CONISOFT'15. Finalmente, invitamos a los lectores de esta Revista especializada en Temas de Ingeniería de Software, a participar en la edición 2016 del Congreso Internacional de Investigación e Innovación en Ingeniería de Software, a celebrarse en la ciudad de Puebla, México, en abril de 2016.

\section{REFERENCIAS}

[1] P. Naur, \& B. Randell. "Software Engineering: Report of a conference sponsored by the NATO Science Committee" Garmisch, Germany, 7-11 Oct. 1968, Brussels, Scientific Affairs Division, NATO. 1969.

[2] J. Buxton \& B. Randell. "Software Engineering Techniques: Report of a conference sponsored by the NATO Science Committee", Rome, Italy, 27-31 Oct. 1969, Brussels, Scientific Affairs Division, NATO. 1970.

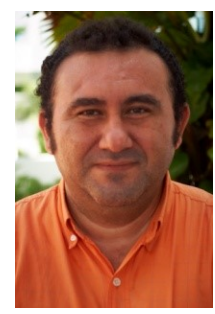

Raúl A. Aguilar Vera posee el Grado de Master en Ingeniería de Software, así como el de Doctor por la Universidad Politécnica de Madrid, España. Es Profesor Titular de tiempo completo en la Facultad de Matemáticas de la Universidad Autónoma de Yucatán. Actualmente es vocero del Cuerpo Académico de Tecnologías para la Formación en Ingeniería de Software. Sus áreas de interés son la Ingeniería de Software y la Informática Educativa.

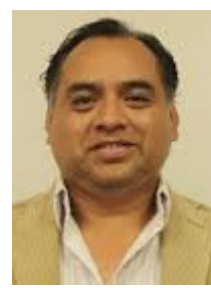

Reyes Juárez Ramírez es Profesor Investigador de la Universidad Autónoma de Baja California. Actualmente preside la Red Temática Mexicana de Ingeniería de Software. Sus principales áreas de interés son la Ingeniería de Software, Interacción Humano-Computadora, Ingeniería de laUsabilidad, Ingeniería del Conocimiento.

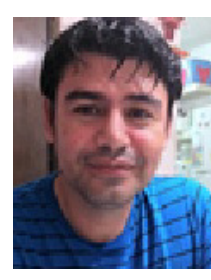

Antonio A. Aguileta Güemez es Licenciado en Ciencias de la Computación por la Universidad Autónoma de Yucatán (UADY), Maestro en Ciencias Computacionales por el Instituto Tecnológico y de Estudios Superiores de Monterrey (ITESM), campus Monterrey. Actual-mente es Profesor Asociado en la Facultad de Matemáticas de la UADY e integrante del Cuerpo Académico de Tecnologías para la Formación en Ingeniería de Software. Su área de interés es la Ingeniería de Software, en particular, temas en el ámbito de Calidad en la Ingeniería de Software. 